\title{
Skeletal muscle mass effects on estimated glomerular filtration rate decrement after donor nephrectomy
}

\author{
Joon Chae $\mathrm{Na}^{1}$, Namki Hong ${ }^{2}$, Min-Gee Yoon ${ }^{1}$, Yumie Rhee ${ }^{2}$, Woong Kyu Han ${ }^{1}$ \\ ${ }^{1}$ Department of Urology, Yonsei University College of Medicine, Seoul, Korea \\ ${ }^{2}$ Division of Endocrinology, Department of Internal Medicine, Yonsei University College of Medicine, Seoul, Korea
}

Background: Although it is well known that skeletal muscle mass influences the level of serum creatinine, the effect of skeletal muscle mass on decreased renal function after nephrectomy is not known. In this study we aimed to evaluate the effect of skeletal muscle mass on estimated glomerular filtration rate (eGFR) calculated by serum creatinine and cystatin C.

Methods: From our kidney donor database, 601 donors were randomly selected in even distribution across age and sex. Skeletal muscle area (SMA) and skeletal muscle density (SMD) was extracted from the L3 level slice on predonation computed tomography scan. Skeletal muscle index (SMI) was calculated by correcting SMA for height: SMA/height ${ }^{2}$. The association between the skeletal parameters and predonation eGFR with decreased eGFR ration at 6-month postdonation compared to predonation was analyzed by linear regression analysis.

Results: Among the selected donors, skeletal muscle parameters were extracted from 592 donors. The skeletal muscle parameters were significantly different according to sex: SMA, $168.0 \pm 23.1 \mathrm{~cm}^{2}$ vs. $109.4 \pm 13.8 \mathrm{~cm}^{2}$; SMD, $45.2 \pm 5.2$ Hounsfield units (HU) vs. $39.5 \pm 6.0 \mathrm{HU}$; and SMI, $57.0 \pm 7.2 \mathrm{~cm}^{2} / \mathrm{m}^{2}$ vs. $43.1 \pm 5.1 \mathrm{~cm}^{2} / \mathrm{m}^{2}$ (all $\mathrm{P}<0.001$ ). All skeletal parameters were significantly associated with age, sex and body mass index. There was a significant correlation between predonation creatinine based eGFR and SMA and also SMI. No correlation was seen between skeletal muscle parameters and cystatin-based eGFR or DTPA (diethyltriaminopentaacetic acid)-measured glomerular filtration rate (GFR). SMA and SMI had negative correlation with the \%change of creatinine based eGFR at 6 -month postdonation in male donors but not in female donors. The association of \%change of cystatin $\mathrm{C}$ based eGFR with all skeletal muscle parameters were not significant.

Conclusions: Male kidney donors with high SMA or SMI are more likely to have decreased creatinine based eGFR after donation. This phenomenon is more likely associated with skeletal muscle mass rather than a reflection of true decrease in GFR. Cystatin $\mathrm{C}$ based eGFR may reflect postdonation GFR more accurately in muscular male donors.

Corresponding author: Woong Kyu Han

E-mail: hanwk@yuhs.ac

(c) The Korean Society for Transplantation

This is an Open Access article distributed under the terms of the Creative Commons Attribution Non-Commercial License (http://creativecommons.org/licenses/by-nc/4.0/) which permits unrestricted non-commercial use, distribution, and reproduction in any medium, provided the original work is properly cited. 